\title{
Determinación de los parámetros hidrodispersivos de una falda acuífera mediante pruebas con trazadores
}

\author{
C. Fallico ${ }^{1}$, M. F. Rivera ${ }^{2 *}$, A. C. Ríos ${ }^{2}$, A. Molinari ${ }^{3}$, P. Santillán ${ }^{2}$ y M. Salazar ${ }^{2}$ \\ ${ }^{1}$ Universidad de Calabria, Departamento de Defensa del Suelo, Puente P. Bucci Cubo 41 B, 87036, Rende, Italia. \\ ${ }^{2}$ Universidad Nacional de Chimborazo, Facultad de Ingeniería, Avenida Antonio José de Sucre, Riobamba EC060104, Ecuador. \\ ${ }^{3}$ Politécnico de Milán, Departamento de Ingeniería Civil y Ambiental, Piazza L. Da Vinci, 32 - 20133 Milán, Italia. \\ *Autor principal/Corresponding author,e-mail: fernanda.rivera@fis.unical.it \\ Editado por/Edited by: Cesar Zambrano, Ph.D. \\ Recibido/Received: 07/05/2013. Aceptado/Accepted: 02/06/2013. \\ Publicado en línea/Published on Web: 28/06/2013. Impreso/Printed: 06/06/2013.
}

\begin{abstract}
During last years the global awareness about human-health and environment risks, caused by aquifers pollution, has grown. In this context, remediation actions on contaminated groundwater has acquired large importance from both scientific and engineering viewpoint whose main aim is the restoration of health-safety values of contaminants in groundwater for human beings. Before to start any remediation action, it is essential to characterize as accurate as possible of the porous medium properties, from both the hydrodispersive and hydrodynamic point of view. Often in situ aquifers characterization requires high economic and human costs and therefore laboratory tests and modelling are usually employed for a first level of characterization. The major aim of this work is to present the results of the main hydrodispersive parameters of a phreatic aquifer artificially reproduced by a laboratory prototype in order to characterize the mass transport within the considered system. Dispersion coefficient and longitudinal dispersivity presented are obtained as a result of a series of tracer tests carried out on the model which allow to complete the hydrodynamic characterization of the reproduced aquifer.
\end{abstract}

Keywords. Aquifer, Hydrodispersive Parameters, Tracers, Dispersion Coefficient, Longitudinal Dispersivity

\section{Resumen}

Durante los últimos años se ha creado una conciencia mundial sobre los riesgos a la salud humana y al medio ambiente, producidos por la contaminación de los acuíferos. En este sentido, las acciones de remediación constituyen un elemento de fuerte interés tanto de un punto de vista científico como ingenierístico, cuyo objetivo fundamental es aquel de reconducir a condiciones seguras los niveles de concentración de los contaminantes encontrados en acuíferos. Sin embargo, antes de proceder a las acciones de remediación en sí, se vuelve fundamental una caracterización lo más precisa posible de este medio poroso, tanto desde el punto de vista hidrodinámico como hidrodispersivo. Los costos tanto económicos como humanos de una caracterización del acuífero in situ muchas veces son insostenibles, por tal motivo, la reproducción en laboratorio de un modelo de acuífero puede ser útil para efectuar un primer nivel de caracterización. Este artículo tiene como finalidad mostrar los resultados obtenidos de los principales parámetros hidrodispersivos de un acuífero freático artificial, reproducido mediante un prototipo de laboratorio, con el objeto de caracterizar el transporte de masa. El estudio se basa en estudios obtenidos de una precedente caracterización hidrodinámica del acuífero en mención. En este estudio se reportan los resultados obtenidos, en el modelo de acuífero, del coeficiente de dispersión y de la dispersividad longitudinal, a través de pruebas de trazabilidad, efectuadas en el prototipo.

Palabras Clave. Acuífero, Parámetros Hidrodispersivos, Trazadores, Coeficiente de Dispersión, Dispersividad Longitudinal

\section{Introducción}

El paso de una economía de tipo agrícola a una de tipo industrial que ha caracterizado las últimas décadas ha dado lugar a nuevos problemas relacionados con la con- taminación ambiental y por ende la necesidad de reparación y recuperación de áreas contaminadas.

La fuerte antropización de algunas zonas, con todo lo que esto implica (zonas industriales, vertederos, etc.) ha llevado a la generación de sustancias, productos y dese- 
chos que se han extendido en diversas matrices ambientales, lo cual constituye un peligro para la salud del hombre y de los ecosistemas con los que entran en contacto [1]. La toma de conciencia de los riesgos asociados con este tipo de situaciones y el desarrollo de nuevos conocimientos científicos, han evidenciado la necesidad de reconducir las zonas afectadas a su estado original y reducir las concentraciones de contaminantes a niveles innocuos, a través de remediación de sitios contaminados mediante la remoción de la causa contaminante y la recuperación de los niveles aceptables de riesgo para la salud humana [2]. Las obras de remediación de sitios contaminados, debido a su complejidad, son un tema de gran interés tanto desde el punto de vista de ingeniería como desde un entorno legal y normativo.

Antes de proceder a la recuperación de un sitio contaminado es indispensable su caracterización con precisión desde el punto de vista hidrodinámico como hidrodispersivo [3]. Esto puede implicar costos considerables, por tal razón una primera experiencia puede ser limitada al estudio del acuífero mediante su reproducción en laboratorio a través de un prototipo y efectuando en este el primer nivel de caracterización.

La finalidad de este trabajo experimental es la de determinar los principales parámetros hidrodispersivos en un acuífero freático artificial, reproducido a través de un modelo físico de laboratorio, con el objeto de caracterizar adecuadamente el transporte de masa. Es importante tener presente que anteriormente se realizó una caracterización hidrodinámica detallada del mismo acuífero dentro en prototipo construido para tal fin.

De manera particular, en este estudio se presentan los resultados de la determinación, para el acuífero artificial en análisis, del coeficiente de dispersión y la dispersividad longitudinal, mediante idóneas pruebas con trazadores realizadas directamente en el modelo físico.

Los resultados presentados a continuación hacen preferencia a pruebas de laboratorio efectuadas con el objetivo de caracterizar desde un punto de vista hidrodispersivo una falda artificial reproducida a escala de laboratorio con la finalidad de estudiar la eficiencia de una barrera reactiva permeable (PRB) en el abatimiento de los metales pesados presentes en el acuífero usando como medio reactivo particulares fibras vegetales. Esta investigación es parte de un acuerdo de cooperación e intercambio entre la Universidad de Calabria (Italia) y la Universidad Nacional de Chimborazo (Ecuador).

\section{Metodología}

\section{Ecuaciones Teóricas para la Caracterización hidrodis- persivas}

La metodología consistió en la caracterización del terreno muestreado en Cosenza, Cosenza, Calabria, Italia desde el punto de vista granulométrico, donde fue verificado el flujo laminar del mismo, determinado mediante

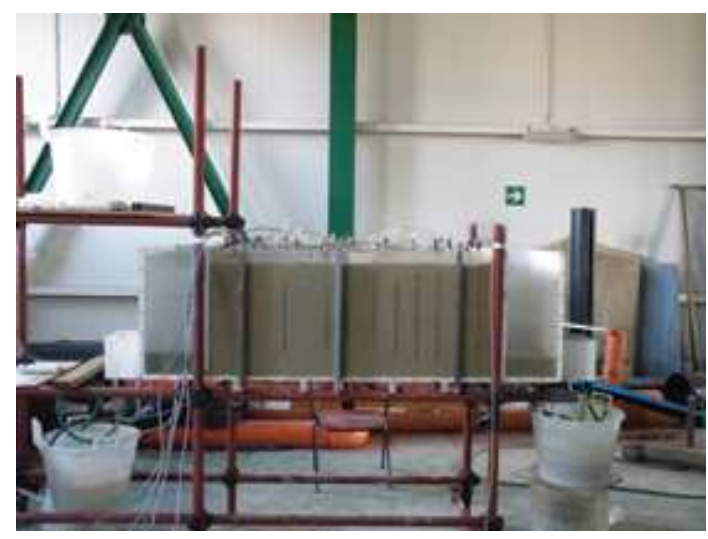

Figura 1: Fotografía del dispositivo utilizado "Sand Box"

el número de Reynolds $R_{e}=\frac{V d}{\nu}$, donde $\mathrm{V}$ es la velocidad efectiva del fluido, d es el diámetro eficaz (magnitud lineal que caracteriza el tamaño de los poros, o de los mismos gránulos sólidos que constituyen la matriz sólida) y $\nu$ es la viscosidad cinemática, a través de este cálculo fue comprobado que el valor de tal parámetro resulta menor que 10 [4].

Desde el punto de vista hidrodinámico del acuífero fue previamente determinando los parámetros hidrodinámicos, en particular la conductividad hidráulica, a través de pruebas de bombeo y el posterior tratamiento de datos experimentales de descensos vs tiempo, obtenidas en condiciones de movimiento no estacionario con el método de Neuman [5].

\section{La caracterización de la hidrodispersividad}

La determinación de los parámetros hidrodispersivos, particularmente el coeficiente de dispersión y la dispersividad, en el presente estudio fueron obtenidas a través de pruebas con trazadores, considerando como trazador el $\mathrm{NaCl}$.

De esta manera, despreciando los fenómenos de decadimento y adsorción - desorción, la ecuación de transporte sólido asume la siguiente expresión [6].

$$
\begin{gathered}
\frac{\partial}{\partial x}\left(D_{x x} \frac{\partial C}{\partial x}\right)+\frac{\partial}{\partial y}\left(D_{y y} \frac{\partial C}{\partial y}\right)-\frac{\partial}{\partial x}\left(u_{1} C\right)+ \\
-\frac{\partial}{\partial y}\left(u_{2} C\right)=S \frac{\partial C}{\partial t}
\end{gathered}
$$

Donde:

$C=$ concentración del contaminante $\left[M L^{-2}\right]\left[M L^{-3}\right]$;

$S=$ coeficiente de almacenamiento [- -]

$D_{x x}=$ coeficiente de dispersión hidrodinámica a lo largo del eje $\mathrm{x}\left[L^{2} T^{-1}\right]$

$D_{y y}=$ coeficiente de dispersión hidrodinámica a lo largo del eje y $\left[L^{2} T^{-1}\right]$

$u_{1}=$ componente de la velocidad a lo largo de $\mathrm{x}\left[L T^{-1}\right]$ 
$u_{2}=$ componente de velocidad a lo largo de y $\left[L T^{-1}\right]$

En la hipótesis de movimiento monodimensional, inmisión impulsiva, superficie libre no significativamente perturbada por la presión generada mediante las pruebas de bombeo, en condiciones de eventual movimiento forzado y dispersión trasversal despreciable, la solución de la Ec. 1 toma la siguiente forma [7].

$$
C(x, t)=\frac{M / \omega}{\sqrt{4 \pi D t}} \cdot e^{-\frac{(x-u t)^{2}}{4 D t}}
$$

Donde:

$M=$ masa del trazador por unidad de sección transversal total $[M]$;

$x=$ distancia entre el punto de inmisión del trazador y el de muestreo $[L]$;

$t=$ tiempo $[T]$;

$\alpha=$ dispersividad $[L]$;

$u=$ velocidad real $\left[L T^{-1}\right]$;

$D=$ coeficiente de dispersión $\left[L^{2} T^{-1}\right]$;

$C=$ concentración en el tiempo t genérico $\left[M L^{-1}\right]$;

$\omega=$ porosidad $[-]$

Si se asumen las siguientes variable reducidas:

$$
\begin{gathered}
t_{R}=\frac{u t}{x} \\
C_{R}=\frac{C(x, t)}{C_{M A X}(x)}
\end{gathered}
$$

Donde:

$C_{M A X}=$ concentración máxima $\left[M L^{-1}\right]$.

La Ec. 2 se convierte en la siguiente:

$$
C_{R}=\frac{K}{\sqrt{t_{R}}} e^{\left[-\frac{P_{e}}{4 t_{R}}\left(1-t_{R}\right)^{2}\right]}
$$

Donde:

$K=\sqrt{t_{R_{M A X}}} \cdot e^{\left[\frac{P_{e}}{{ }^{t} R_{M A X}}\left(1-t_{R_{M A X}}\right)^{2}\right]}$

$t_{R_{M A X}}=\sqrt{1+\left(\frac{1}{P}\right)^{2}}-\frac{1}{P_{e}}$

$P_{e}=$ número de Péclet [-]

Donde $P_{e}=\frac{V_{e f} x}{D}$

Sustituyendo en la Ec.3 los diferentes valores del número de Péclet, se obtienen las curvas teóricas que sobreponen la experimental, la identificación de la curva teórica es representativa del caso en estudio y se determina así los valores del coeficientes dispersión (D) y la dispersividad $(\alpha)$ [8-12] definida como $\alpha=\frac{x}{P_{e}}$.

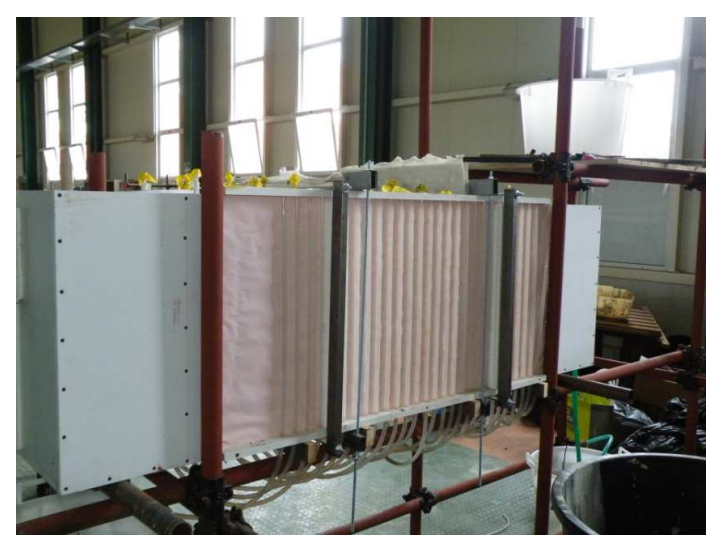

Figura 2: Sistema para la lectura de los niveles piezométricos

En cuanto concierne la velocidad de transporte en el interior del medio poroso, después de haber determinado un flujo laminar y estacionario, la velocidad se obtiene a partir de la ley de Darcy:

$$
V_{D}=-k \frac{\Delta h}{L}
$$

mientras que la velocidad efectiva se determina mediante la siguiente ecuación $[4,9]$ :

$$
v_{e f}=\frac{\int_{0}^{\infty} \frac{x}{t} C(t) d t}{\int_{0}^{\infty} C(t) d t}=x \frac{I_{3}}{I_{2}}
$$

Donde:

$t=$ tiempo $[\mathrm{T}]$;

$C(t)=$ concentración del contaminante en el tiempo $\mathrm{t}$ $\left[M L^{-2}\right]$;

$I_{3}=\int_{0}^{\infty} \frac{1}{t} C(t) d t$

$I_{2}=\int_{0}^{\infty} C(t) d t$

$V_{D}$ y de $V_{e f}$ se pueden utilizar para determinar la porosidad del medio estudiado mediante la siguiente ecuación $[4,9]$ :

$$
\omega=\frac{V_{D}}{V_{e f}}
$$

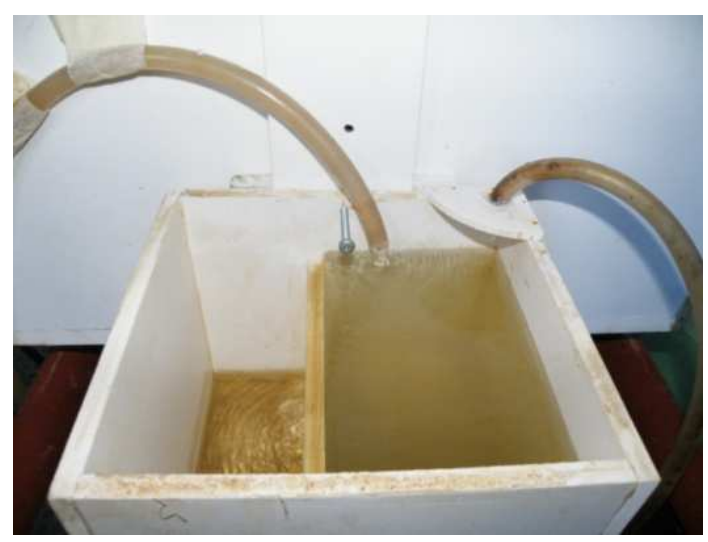

Figura 3: Sistema de bandejas móviles para regularizar la carga 


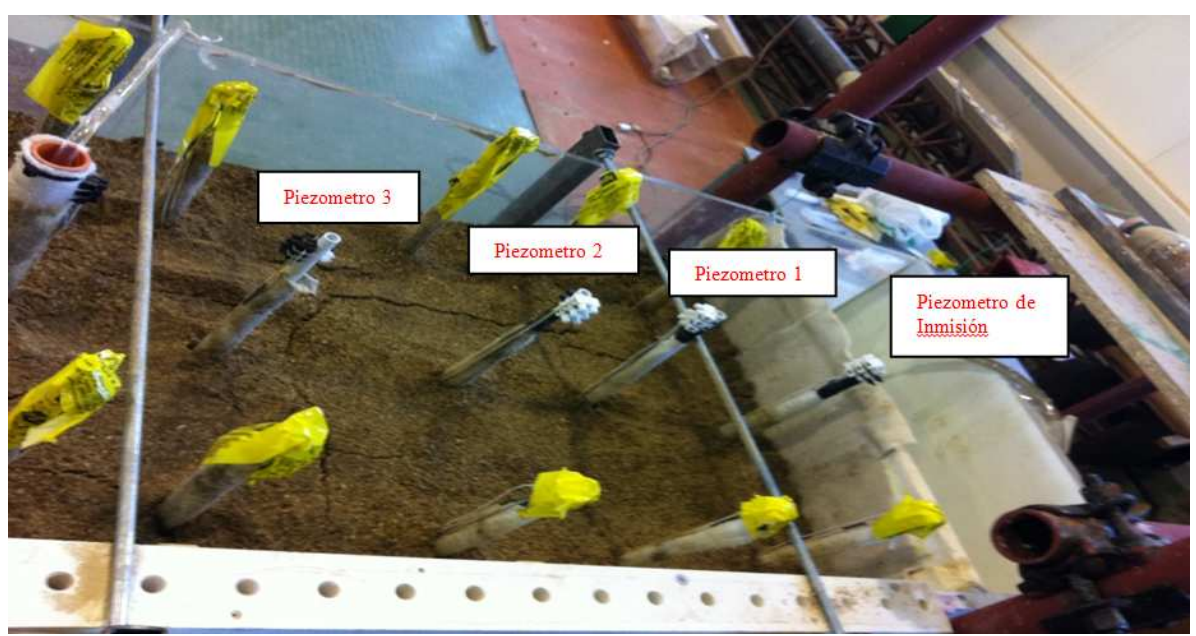

Figura 4: Dispositivo usado para la prueba con trazadores, con pozo de bombeo, piezómetro de inmisión, y pozos de observación.

\section{Modelo experimental}

La fase experimental se llevó a cabo en un acuífero subterráneo artificial especialmente diseñado mediante un modelo físico situado en el Laboratorio de Defensa del Suelo de la Universidad de Calabria (Italia) y exactamente reproducido en el Laboratorio de Servicios Ambientales de la Universidad Nacional Chimborazo (Ecuador). Este modelo consiste en una caja en forma de paralelepípedo de dimensiones $0,60 \mathrm{~m} \times 2 \mathrm{~m} \times 0,60 \mathrm{~m}$, descubierto en la parte superior y colocado a $1 \mathrm{~m}$ a partir de la superficie suelo sostenido con una estructura metálica con tubos de conexión.

La parte posterior y los dos paneles laterales están hechos de PVC (cloruro de polivinilo), del espesor de 2 $\mathrm{cm}$, mientras que la parte delantera es hecha de una lámina de plexiglás transparente de espesor $1 \mathrm{~cm}$ que permite la inspección visual del experimento.

En correspondencia de las dos extremidades del paralelepípedo han sido colocadas dos tanques cuya altura se puede regular para fijar la gradiente de la carga hidráulica entre los dos extremos, fijando de esta manera las condiciones de flujo al interno del modelo. Al interno de la caja están presentes una serie de piezómetros aforados recubiertos de un geotextil usado para impedir la obstrucción de los foros por parte de la arena colocada dentro de la caja.

Una fotografía del dispositivo en cuestión se muestra en la Figura 1.

El modelo fue equipado con veinte y siete piezómetros, dispuestos a lo largo de tres filas de nueve, que consisten en tubos de plástico rígido de largo $0.60 \mathrm{~m}$, de diámetro $0.008 \mathrm{~m}$, perforado a lo largo de toda su longitud con una distancia entre ejes de $0.05 \mathrm{~m}$ y recubiertos con el mismo geotextil utilizado en las paredes del modelo. Cada piezómetro, fue instalado en el interior de un agujero hecho en la parte inferior de la caja de arena, y conectado al exterior del modelo con un tubo de plástico transparente y flexible, con el cual es posible determinar la carga hidráulica, midiendo el nivel de agua en los tubos de plástico transparentes dispuestos en la parte posterior del modelo, por encima de una hoja de papel cuadriculado, como se indica en la Figura 2.

En el compartimiento central ha sido realizado un pozo, a través de un tubo de PVC con un diámetro de 0.030 $\mathrm{m}$, el cual está también cubierto por geotextil, con orificios realizados cada $0.03 \mathrm{~m}$ a lo largo de toda su profundidad. Este pozo está ubicado en el centro de la caja, con el fin de tener el mismo número de piezómetros en ambos lados y, por lo tanto, en posición útil cualquiera que sea la dirección del flujo de agua. Para garantizar una carga constante en los tanques laterales, se ha predispuesto un sistema de bandejas móviles conectadas al modelo. Cada una de las bandejas móviles, que sirve para asegurar la carga constante en los compartimientos laterales, tienen la capacidad de deslizarse verticalmente a lo largo de un carril fijado en la pared de los tanques de carga y descarga y se divide en dos compartimentos, uno de los cuales, conectado hidráulicamente con el sistema, viene alimentado continuamente y está equipado con un rebosadero para mantener un nivel de agua constante en el interior, como se indica en la Figura 3 .

Para la caracterización hidrodispersiva, se hizo una prueba de trazadores con movimiento forzado, bombeando desde el pozo central con un caudal constante de 0.838 $\mathrm{m}^{3} / \mathrm{d}$, con ayuda de bomba peristáltica de pequeña potencia. Utilizando para la inmisión del trazador el piezómetro derecho más lejano del pozo. Se prepararon $100 \mathrm{ml}$ de una solución de $\mathrm{NaCl}$ de $80 \mathrm{~g} / \mathrm{l}$, que fue inyectada en el piezómetro en cuestión en un corto tiempo [13]. Sucesivamente, la prueba es de tipo continuo realizando lec-

\begin{tabular}{cc}
\hline \multicolumn{2}{c}{ Distribución granulométrica } \\
\hline Tipo de Suelo & $\%$ \\
\hline Grava & 17.5 \\
Arena & 82.5 \\
Fango & 0.0 \\
Arcilla & 0.0 \\
\hline
\end{tabular}

Tabla 1: Resultados de la distribución granulométrica del terreno 


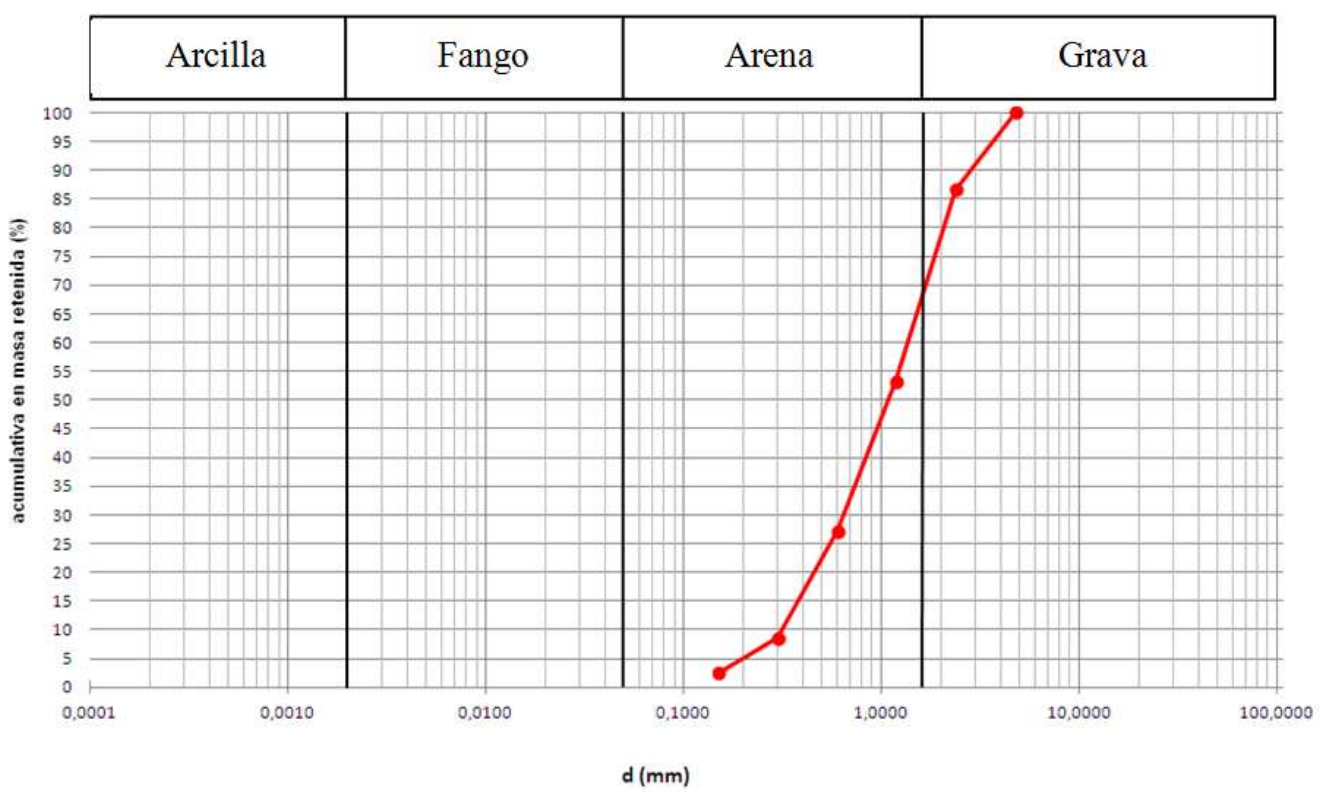

Figura 5: Clasificación granulométrica del terreno en estudio

turas de conductividad eléctrica, por medio de un conductivimetro Modello HI 9033 Multi-range, y determinando a través de este los correspondientes valores de concentración del trazador, desde las muestras tomadas, cada vez, en los 3 piezómetros de medición alineados en el modelo, por medio de una jeringa provista de una tubo de goma especialmente preparado [14]. Los pozos de bombeo y los pozos de observación se muestran en la Figura 4.

Los piezómetros de observación n.1, n. 2 y n. 3 están alejados del piezómetro de inmisión $0.15 \mathrm{~m}, 0.30 \mathrm{~m}$ $0.45 \mathrm{~m}$ respectivamente.

Verificada la laminaridad y la estacionariedad de movimiento $(\operatorname{Re} \cong 0,2<10)$, mediante la Ec.4 se determina la velocidad de Darcy $V_{D}$ y mediante la ecuación (5) la velocidad efectiva $V_{e f}$. Finalmente, mediante la Ec.6, se obtiene la porosidad del medio.

Sucesivamente se repitió la prueba con el trazador con una caudal, igual a $0.616 \mathrm{~m}^{3} / \mathrm{d}$. Seguidamente fue modificado el espesor de la capa freática, llevándola a 0.282 $\mathrm{m}$, y luego fueron repetidas las pruebas con el trazador con los mismos dos caudales asumidos anteriormente, es decir, $0.838 \mathrm{~m}^{3} / \mathrm{d}$ y $0.616 \mathrm{~m}^{3} / \mathrm{d}$.

\section{Análisis granulométrico}

El terreno utilizado (medio poroso) fue sometido a un análisis granulométrico cuyos resultados son presentados en la Tabla 1, mientras que la Figura 5 muestra la curva granulométrica correspondiente. Estos resultados permiten definir el medio poroso considerado como arena.

El compartimiento central del modelo fue llenado con tal terreno a una altura de aproximadamente $0.50 \mathrm{~m}$ y se ha hecho una inmisión de agua para simular un acuífero freático a una altura de aproximadamente $0.408 \mathrm{~m}$, sucesivamente se repitió la prueba con una altura de la falda de $0.282 \mathrm{~m}$.

\section{Resultados y Discusión}

Después de la caracterización hidrodinámica del terreno para la estimación de la conductividad hidráulica mediante pruebas de flujo se ha obtenido un valor de $1.27 \mathrm{x}$ $10^{-6} \mathrm{~m} / \mathrm{s}$.

Los valores de la velocidad efectiva de Darcy y la porosidad, obtenidos en correspondencia de cada piezómetro, son reportan en la Tabla 2.

El muestreo efectuado en cada uno de los piezómetros ha hecho posible obtener las curvas de restitución para cada uno de los casos examinados, es decir distintos espesores del acuífero y diversos caudales de bombeo. En la Figura 6 se muestran las curvas de restitución en términos de variables reducidas para cada piezómetro y para cada una de las hipótesis formuladas.

Para cada uno de las pruebas de trazabilidad realizadas se ha efectuado la superposición con las curvas teóricas obtenidas cambiando el número de Péclet con la ecuación (4), válido en el caso de un movimiento uniforme de una dimensión y colocación impulsivo. En la Figura 7 se muestran los gráficos de prueba para cada uno de rastreo con superposiciones con la finalidad de poder determinar el valor del número de Peclet $\left(P_{e}\right)$ para cada una de las pruebas de trazabilidad realizadas, relativas a las diversas configuraciones consideradas en el sistema experimental.

Para encontrar el valor del número de Peclet, y por lo tanto la curva teórica que mejor se sobrepone con la curva experimental, se procedió tomando como valor de 


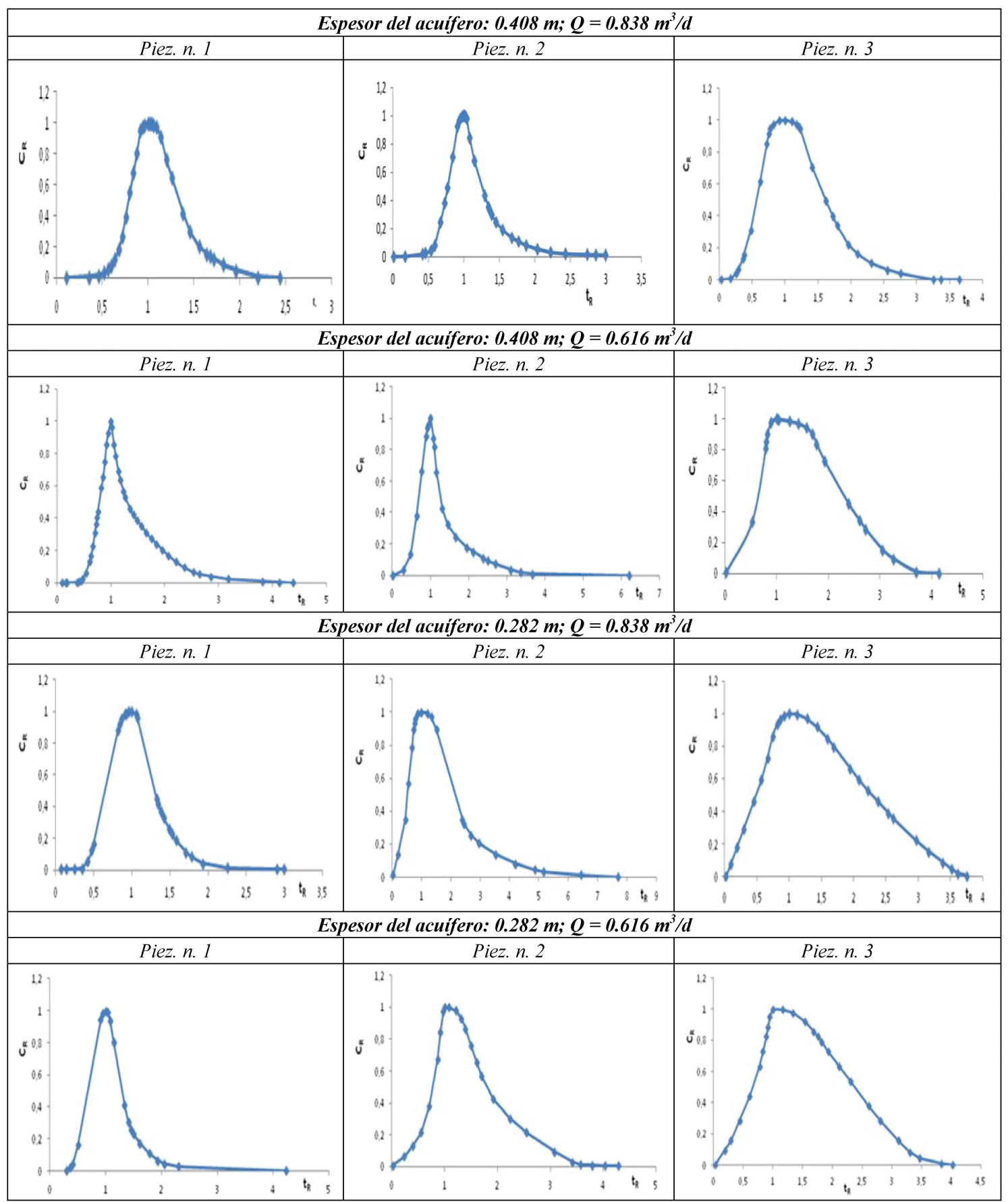

Figura 6: Curvas de restitución obtenidas en los tres piezómetros en estudio, para cada uno de los dos espesores del acuífero y para cada uno de los caudales de bombeo considerados

dicho parámetro aquel que minimiza el error de superposición entre las dos curvas. Para hacer este método se adoptó el método de error estándar $(S E)$, que describe la incertidumbre en la estimación de un valor estadístico. $S E$ es por lo tanto, en este caso, una estimación de la variabilidad de la desviación estándar de los parámetros considerados relativos a la muestra, una medida de la imprecisión de las variables reducidas $C_{R}$ y $d t_{r}$ se obtiene como diferencia en valor absoluto entre los valores de estos parámetros relacionados con la curva experimental y la teórica [15]. El error estándar se representa mediante la siguiente expresión [16]:

$$
S E=\frac{\sigma}{\sqrt{n}}
$$

Donde:

$\sigma=$ desviación estándar de la población;

$n=$ número de valores considerados en la muestra (hasta el valor máximo de $C_{R}$ ). En la Figura 8 se muestran para mayor claridad del gráfico $S E-P_{e}$ relativo 


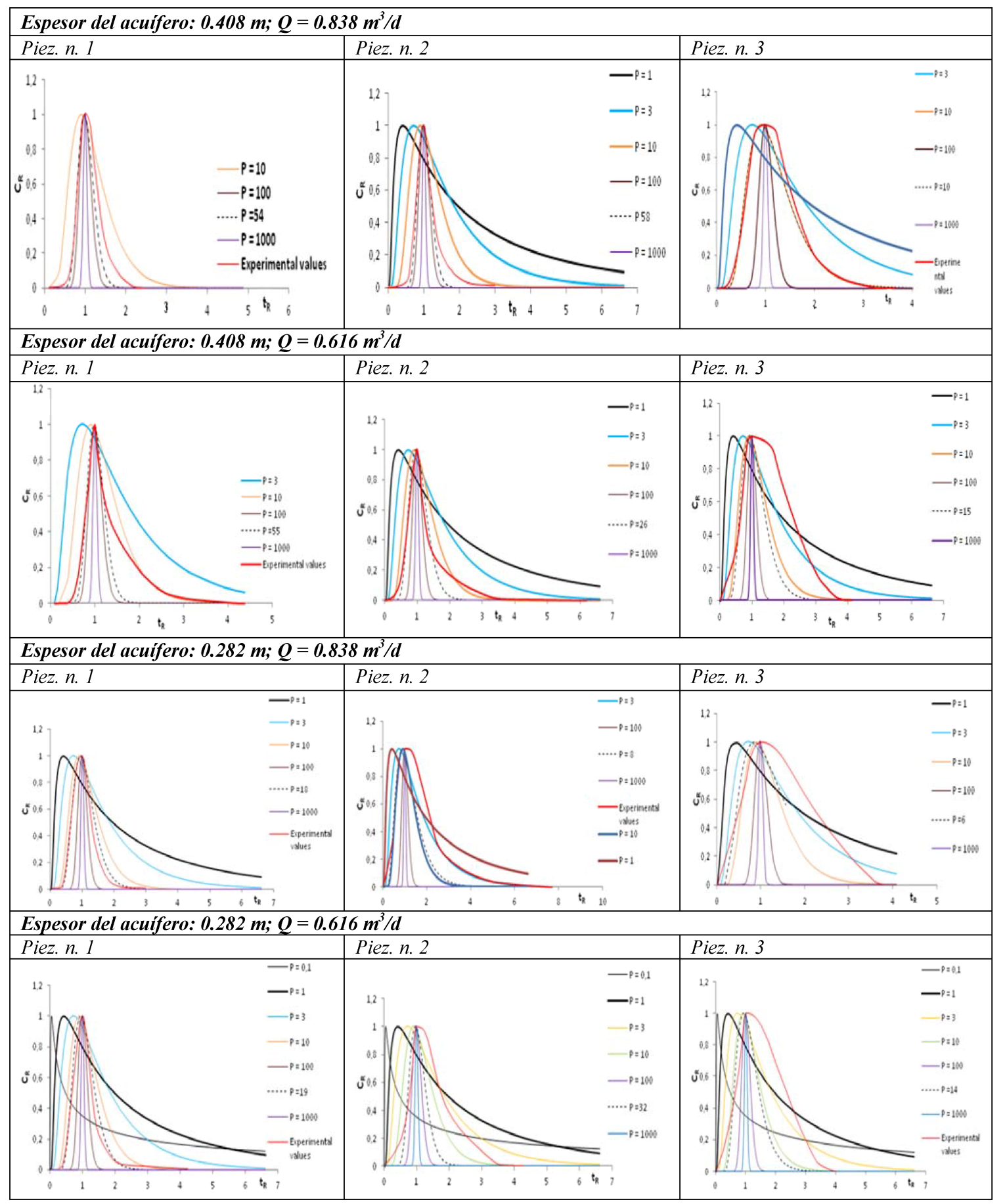

Figura 7: Superposición de las curvas experimentales con las teóricas para los tres pozos en estudio, para cada uno de los dos grosores del acuífero y para cada de los dos caudales de bombeo considerados

al piezómetro No. 1 y a la prueba de trazadores realizada con el espesor del acuífero igual a $0.408 \mathrm{~m}$ y caudal $0.616 \mathrm{~m}^{3} / \mathrm{s}$.

Recordando que: $P_{e}=\frac{V_{e f} x}{D}$, donde Vef es la velocidad efectiva y x la distancia entre el punto de inmisión y el de observación y D el coeficiente de dispersión, se calcula el último parámetro mediante la siguiente expresión:

$$
D=\frac{V_{e f} x}{P_{e}}
$$

Análogamente, se recuerda que siendo $D=V_{e f} \cdot \alpha$, la expresión de dispersividad es la siguiente:

$$
\alpha=\frac{x}{P_{e}}
$$

Mediante las ecuaciones (9) y (10) se determinaron, los 


\begin{tabular}{c|c|c|c|c|c}
\hline Espesor de la falda acuífera (m) & Piezometro & $\mathbf{Q}\left(\mathbf{m}^{\mathbf{3}} / \mathbf{d}\right)$ & $\mathbf{V}_{\mathbf{D}}(\mathbf{m} / \mathbf{d})$ & $\mathbf{V}_{\text {eff }}(\mathbf{m} / \mathbf{d})$ & $\omega$ \\
\hline \multirow{5}{*}{$0.408 \mathrm{~m}$} & $\begin{array}{l}\mathrm{n}^{\circ} 1 \\
\mathrm{n}^{\circ} 2 \\
\mathrm{n}^{\circ} 3\end{array}$ & 0.838 & 3.423 & 4.507 & 0.759 \\
\cline { 2 - 6 } & $\begin{array}{l}\mathrm{n}^{\circ} 1 \\
\mathrm{n}^{\circ} 2 \\
\mathrm{n}^{\circ} 3\end{array}$ & 0.616 & 2.516 & 3.202 & 0.785 \\
\hline \multirow{5}{*}{$0.282 \mathrm{~m}$} & $\begin{array}{l}\mathrm{n}^{\circ} 1 \\
\mathrm{n}^{\circ} 2 \\
\mathrm{n}^{\circ} 3\end{array}$ & 0.838 & 4.963 & 6.359 & 0.780 \\
\cline { 2 - 6 } & $\begin{array}{l}\mathrm{n}^{\circ} 1 \\
\mathrm{n}^{\circ} 2 \\
\mathrm{n}^{\circ} 3\end{array}$ & 0.616 & 3.717 & 4.497 & 0.826 \\
\hline
\end{tabular}

Tabla 2: Valores de $V_{D}, V_{\text {eff }} \mathrm{y} \omega$ en los piezómetros, para los diversos espesores de la falda en los caudales considerados

\begin{tabular}{c|c|c|c|c|c}
\hline Espesor de la falda acuífera (m) & Piezometro & $\mathbf{Q}\left(\mathbf{m}^{\mathbf{3}} / \mathbf{d}\right)$ & $\mathbf{P}_{\mathbf{e}}$ & $\mathbf{D}\left(\mathbf{m}^{\mathbf{2}} / \mathbf{s}\right)$ & $\alpha(\mathbf{m})$ \\
\hline \multirow{5}{*}{$0.408 \mathrm{~m}$} & $\mathrm{n}^{\circ} 1$ & & 54 & 0.024 & 0.003 \\
& $\mathrm{n}^{\circ}$ & 0.838 & 58 & 0.062 & 0.005 \\
& $\mathrm{n}^{\circ} 3$ & & 10 & 0.680 & 0.045 \\
\cline { 2 - 6 } & $\mathrm{n}^{\circ} 1$ & & 55 & 0.017 & 0.003 \\
& $\mathrm{n}^{\circ}$ & 0.616 & 26 & 0.115 & 0.011 \\
& $\mathrm{n}^{\circ} 3$ & & 15 & 0.436 & 0.030 \\
\hline \multirow{5}{*}{$0.282 \mathrm{~m}$} & $\mathrm{n}^{\circ} 1$ & & 18 & 0.070 & 0.008 \\
& $\mathrm{n}^{\circ}$ & 0.838 & 8 & 0.450 & 0.037 \\
& $\mathrm{n}^{\circ} 3$ & & 6 & 1.202 & 0.075 \\
\cline { 2 - 6 } & $\mathrm{n}^{\circ} 1$ & & 19 & 0.094 & 0.008 \\
& $\mathrm{n}^{\circ} 2$ & 0.616 & 32 & 0.148 & 0.009 \\
& $\mathrm{n}^{\circ} 3$ & & 14 & 0.781 & 0.032 \\
\hline
\end{tabular}

Tabla 3: Valores del número de Peclet $\left(\mathrm{P}_{\mathrm{e}}\right)$, del coeficiente de dispersión y de la dispersividad para las pruebas con trazadores efectuadas

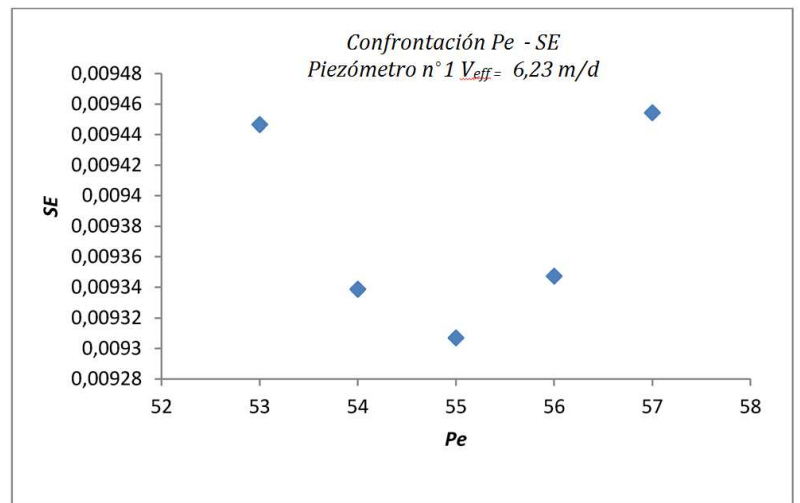

Figura 8: Individualización del valor de $P_{e}$ al cual correspondiente al valor mínimo de $S E$ (Piez. n.1, espesor del acuífero $\mathbf{H}=$ $0.408 \mathrm{~m}, \mathrm{Q}=0.616 \mathrm{~m}^{3} / \mathrm{s}$ )

valores de $\mathrm{D}$ y $\alpha$ para cada una de las pruebas con trazadores y de las configuraciones del sistema tomado en consideración, en la Tabla 3 se presentan los valores de estos parámetros.

\section{Conclusiones}

La realización de las pruebas con trazadores en un acuífero freático artificial, reproducido en laboratorio utilizando un modelo físico, ha permitido la caracterización hidrodispersivas de un trazador (sal), considerando como medio poroso identificado después de los análisis de laboratorio la arena. En esta investigación se han determinan- do los parámetros hidrodispersivos en diferentes condiciones del nivel de la falda, en particular, se han obtenido el coeficiente de dispersión y la dispersividad.

La caracterización hidrodispersiva del acuífero artificial, en estudio, representa el punto de partida para posibles actividades de investigación futuras sobre los aspectos vinculados al transporte de masa en los medios porosos, con la finalidad de individuar las mejores técnicas de remediación para los acuíferos contaminados.

En conclusión, los resultados obtenidos muestran como la caracterización hidrodinámica efectuada en escala de laboratorio para el acuífero artificial estudiado (que representa una simplificación de los acuíferos reales) representan un instrumento válido para la comprensión de los fenómenos de flujo y transporte que se podrían verificar en escala real.

\section{Referencias}

[1] Swoboda - Colberg, N. 1995. "Chemical contamination of the environment: sources, types and fate of synthetic organic chemicals". Microbial transformation and degradation of toxic organic chemicals. Wiley - Liss Inc.

[2] Ministero dell' Ambiente Italiano. 2006. Norme in materia ambientale. Decreto Legislativo n. 152 del 3 aprile 2006. Decreto Legislativo n. 152 del 3 aprile 2006, [D.lgs. 152/06].

[3] Hamill, L.; Bell, F. 1986. "Ground water resource development”. Butterworth Heinemann Ltd. Oxford. 
[4] Bear, J. 1979. "Hydraulics of Groundwater". McGrawHill. New York.

[5] Freeze, R.; Cherry, J. 1979. "Groundwater". Prentice Hall Ed.

[6] Batu, V. 1998. "Aquifer Hydraulics: A Comprehensive Guide to Hydrogeologic Data Analysis". Wiley.

[7] Sauty, J. 1978. "Identification des paramètres du transport hydrodispersif dans les aquifères par interprétation de traçages en ecoulement cylindrique convergent ou divergent". Journal of hydrology, 39.

[8] Bear, D.; Beljin, M.; Ross, R. 1992. "Fundamentals of ground-water modeling". Superfund Technology Support Center for Ground Water.

[9] Troisi, S.; Fallico, C.; Coscarelli, R.; Caramuscio, P. 1992. "Considerazioni sulle misure sperimentali dei parametri idrodispersivi di falde sotterranee". Proceedings of XXIII Convegno di Idraulica e Costruzioni Idrauliche. Firenze, 31 agosto - 4 settembre.

[10] Issar, A.; Brown, N. 1998. "Water, Environment and Society". Times of Climatic Change.

[11] Custodio, E.; Llamas, M. 2001. "Hidrologia Subterranea". Ed. Omega, $2^{a}$ ed.

[12] Celico, P. 1986. "Prospezioni Idrogeologiche". Liguori Ed., Napoli, 1.

[13] Neuman, S. 1972. "Theory of flow in unconfined aquifers considering delayed response of the water table". Water Resour. Res, 8:1031 - 1045.

[14] Xu, M.; Eckestein, Y. 1997. "Statistical Analysis of the relationships between dispersivity and other Physical properties of porous media". 5 .

[15] Devore, J.; Famum, N.; Doi, J. 2013. “Applied Statistics for Engineers and Scientists". 3.

[16] Troisi, S.; Fallico, C.; Straface, S.; Mazzuca, L. 2008. "Biodreni per la bonifica di siti contaminati realizzati con fibre naturali liberiane ad elevato sviluppo superficiale". 